\title{
Solar Cycle Dependence of Spacecraft Charging in Low Earth Orbit
}

\author{
T. B. FROONINCKX ${ }^{1}$ AND J. J. SOJKA \\ Center for Atmospheric and Space Sciences, Utah State University, Logan
}

\begin{abstract}
Recent experimental evidence has shown that Defense Meteorological Satellite Program (DMSP) polar orbiting spacecraft at $840 \mathrm{~km}$ can develop electric potentials as severe as $-1430 \mathrm{~V}$ while at high magnetic latitudes. To explore this charging region, an analysis of DMSP F6, F7, F8, and F9 satellite precipitating particle and ambient plasma measurements taken during periods of high, medium, and low solar flux is performed. One hundred eighty-four charging events ranging from -46 to $-1430 \mathrm{~V}$ are identified, and an extreme solar cycle dependence is found as charging is most frequent and severe during solar minimum. Satellite measurements and time-dependent ionospheric model (TDIM) output are used to determine the cause of the solar cycle dependence and to characterize the environments which generate and inhibit these potentials. The electron precipitation associated with various DMSP charging levels is analyzed; it is suggested that precipitating electrons as low as 2 to $3 \mathrm{keV}$ may contribute to charging though higher-energy electrons make greater contributions. Secondary electron production due to incident electrons below $1 \mathrm{keV}$ is shown to inhibit charging. The energetic electron fluxes shown to generate charging do not vary significantly over the solar cycle. Instead, DMSP ambient plasma data and TDIM generated results identify a variation in plasma density over 1 or more orders of magnitude as the cause of the solar cycle dependence, and an ambient plasma density of less than $10^{4} \mathrm{~cm}^{-3}$ is found necessary for significant negative charging $(\geq 100 \mathrm{~V})$ to occur.
\end{abstract}

\section{INTRODUCTION}

The discovery of multikilovolt negative spacecraft-toplasma potential differences in geosynchronous orbit [DeForest, 1972] and the realization that spacecraft charging often leads to serious operational anomalies [Rosen, 1976] led the scientific community to aggressively pursue an understanding of the environments which generate large electric potentials. In building a theoretical foundation, Whipple, Parker, Laframboise, and others (see review by Garrett [1981]) have provided for an understanding of the plasma sheaths surrounding a charged spacecraft as well as plasma wake and $\vec{V} \times \vec{B}$ effects. Experimentally, charging levels as severe as $-19,000 \mathrm{~V}$ in eclipse and $-2000 \mathrm{~V}$ in sunlight have occurred within the low-density plasma sheet during enhanced fluxes of energetic electrons [Olsen, 1987], and geosynchronous spacecraft potentials such as these typically correlate with intense fluxes of electrons with energies greater than 10 to $30 \mathrm{keV}$ [Olsen, 1983; Gussenhoven and Mullen, 1983; Mullen et al., 1986]. Though ambient plasma can play a key role in inhibiting negative charging by supplying a neutralizing ion current, low thermal plasma densities $\left(\leq 1 \mathrm{~cm}^{-3}\right)$ at geosynchronous altitudes cannot prevent frequent and severe charging. Since plasma densities within the upper ionosphere are normally much greater $\left(10^{2}\right.$ to $10^{5} \mathrm{~cm}^{-3}$ ), spacecraft charging within low Earth orbit (LEO) is commonly thought to be less significant. However, theory predicts negative charging levels as severe as several kilovolts for large structures in polar LEO [Katz and Parks, 1983], and manned polar flights are believed to face additional hazards [Hall et al., 1987]. Measurements in LEO are necessary to better characterize the charging environment (sheath asymmetries) and to identify the key electrodynamic processes [Samir, 1983].

\footnotetext{
${ }^{1}$ Now at the Air Force Space Forecast Center, Falcon Air Force Base, Colorado.

Copyright 1992 by the American Geophysical Union.

Paper number $91 \mathrm{JA} 02704$.

0148-0227/92/91JA-02704\$05.00
}

Previous experimental evidence shows that Defense Meteorological Satellite Program (DMSP) polar orbiting spacecraft can develop electric potentials as large as $-680 \mathrm{~V}$ with respect to their high-latitude "thin" plasma sheath 840 $\mathrm{km}$ environment [Gussenhoven et al., 1985]. We extend their study and investigate a charging-to-solar cycle dependence while analyzing the environments which generate and inhibit high-latitude upper ionospheric charging. Specifically, the primary causes, frequency, severity, and solar cycle dependence of space vehicle charging are identified using extensive DMSP satellite measurements taken during different periods in the solar cycle. Measured precipitating electron fluxes and ambient plasma densities during times of charging and noncharging are closely analyzed to identify key processes and critical values, and ionospheric model plasma densities are used to further characterize the solar cycle variation of ambient plasma density.

To begin, the results of Gussenhoven et al. [1985] are reviewed in section 2 and serve as a quantitative foundation for this study. Section 3 describes the data base and analysis procedures and includes descriptions of the DMSP satellites' orbits and instruments. The results of charging for solar minimum, medium, and maximum conditions are given in section 4 , and a discussion is presented in section 5. Finally, conclusions are listed in section 6.

\section{Previous Study of High-LatTtude LEO Charging}

Gussenhoven et al. [1985] performed a partial survey of northern hemispheric DMSP F6 and F7 precipitating particle and ambient plasma measurements taken during January, November, and December 1983 and identified 11 negative charging events ranging from -47 to $-670 \mathrm{~V}$ and lasting 2 to 62 sec. The electric potential between the vehicle and local plasma exceeded $-100 \mathrm{~V}$ only when the satellite encountered intense and energetic magnetic field-aligned electron precipitation while in a region of locally depleted thermal plasma density within the Earth's shadow. Charging levels often ranged over hundreds of volts from one second to the 
next. A positive correlation was found between the charging magnitudes and the ratios of the high-energy ( $\geq 14 \mathrm{keV})$ electron integral number fluxes to the ambient plasma densities; little correlation was found between charging levels and total electron number fluxes, and relationships of charging to electron fluxes other than $\geq 14 \mathrm{keV}$ were not investigated. The $\geq 14 \mathrm{keV}$ electron fluxes typically exceeded $10^{8}$ electrons $/\left(\mathrm{cm}^{2} \mathrm{sec}\right.$ ster $)$, and the ambient plasma densities simultaneously ranged from $10^{1}$ to $10^{4} \mathrm{~cm}^{-3}$. Charging occurred between $68^{\circ} \mathrm{N}$ and $78^{\circ} \mathrm{N}$ magnetic latitude and 2000 and 0230 magnetic local time and was located on the poleward edge of a region of discrete auroras. A study of the electron spectra generating these DMSP charging events was subsequently performed to assist efforts in modeling the charging of large structures in polar orbit.

Yeh and Gussenhoven [1987] evaluated the kilovolt precipitating electron profiles that resulted in the 11 charging events reported by Gussenhoven et al. [1985]. They classified $92 \%$ of the spectra into four groups, parameterized the spectra, and presented empirical values of parameters which could be used for the modeling of spacecraft charging. They used their modeled electron distributions and two sets of secondary electron and backscatter yield coefficients for several materials to calculate critical energies $\left(E_{c}\right)$ above which the electron flux defines the net charging current. Their results for kapton, the primary material of DMSP satellites, showed positive correlations between observed negative charging levels and the ratios of the measured electron number fluxes above $E_{c}$ (unique to each model electron spectral group) to the ambient plasma densities. Though their modeling efforts met with some success, both the critical energies and electron fluxes above $E_{c}$ necessary to produce the observed charging levels vary significantly depending on the secondary and backscatter coefficients used. The models are further limited by unique spectral types which were developed based on 11 charging events. Since the study of Gussenhoven et al. [1985] was a partial data survey, the frequency and magnitude of polar LEO charging have yet to be fully documented.

\section{Data Base}

\subsection{Orbits and Instrumentation}

The DMSP spacecraft are a series of satellites in circular polar orbits with altitudes near $840 \mathrm{~km}$, orbital inclinations of $98^{\circ}$, and periods of approximately $101 \mathrm{~min}$. Two satellites are normally in Sun-synchronous orbit at one time; one satellite orbits in the 0600-1800 (dawn to dusk) local time (LT) meridian, and the other satellite orbits in the 1030-2230 LT meridian. The four satellites which supplied data for this study are designated as F6 and F8 (0600-1800 LT) and F7 and F9 (1030-2230 LT). The F6 was launched in December 1982 and was replaced by the F8 in June 1987, and the F7 was launched in November 1983 and was replaced by the F9 in February 1988. The satellites are nearly the same in material and design, and in-flight cross calibration has been performed such that a comparison of the satellites' charging may be accomplished. With two satellites in different polar orbits at the same time the high-latitude coverage is appreciable.

Since the geomagnetic and geographic poles are offset, the Sun-synchronous DMSP spatial coverage in magnetic local time and geomagnetic latitude includes a significant part of the auroral and polar cap regions. Figure 1 illustrates the two

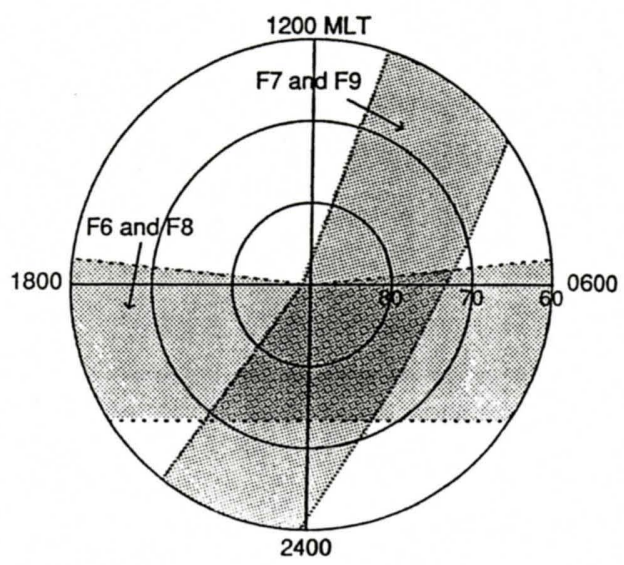

Fig. 1. Orbital coverage of the DMSP F6, F7, F8, and F9 satellites in corrected geomagnetic latitude and magnetic local time over the highlatitude northern hemisphere.

satellite combined coverage over the high-latitude northern hemisphere. Satellite charging can be identified and the in situ environment generating the charging can be analyzed using the DMSP satellites' precipitating particle and thermal plasma detectors.

The precipitating particle detector (SSJ/4) measures the flux of precipitating electrons and ions having energies between 30 and $30,000 \mathrm{eV}$. A detector consists of four curved plate electrostatic analyzers arranged in two pairs; one pair measures electrons while the second pair measures ions. The detectors' apertures always face radially outward from the Earth, so only precipitating electrons/ions rather than ambient or backscattered particles are measured at high magnetic latitudes. One electron/ion analyzer measures over the energy range from $30 \mathrm{eV}$ to $1 \mathrm{keV}$ via 10 channels, while the other measures from 1 to $30 \mathrm{keV}$ via 10 channels, and each channel dwells for nearly $0.1 \mathrm{sec}$; a complete 20 -point electron and ion spectrum is reported once per second. Details and calibration of the SSJ/4 are given by Hardy et al. [1984] and Schumaker et al. [1988]. DMSP F8 and F9 instrumentation also includes a thermal plasma detector.

The special sensor for ions, electrons, and scintillation (SSIES) flown on the F8 and F9 satellites measures the ambient plasma. The sensor consists of a planar ion retarding potential analyzer to determine the densities of different ion species, a planar ion density trap (scintillation meter) to measure the total ambient ion density and its fluctuations, a planar ion drift meter to measure the bulk flow velocity of the thermal plasma, and a spherical Langmuir probe to measure the ambient electrons. A detailed description of the instrument is given by Greenspan et al. [1986]. The F8 and F9 ambient plasma density measurements are taken from the scintillation meter. Though an instrument similar to the SSIES flew on the F6 and F7 satellites, it was inoperable during the period of this study (F. J. Rich, private communication, 1990). The SSJ/4 and SSIES measurements form this study's data base.

\subsection{Data and Analysis}

The solar maximum data base consists of $\mathrm{SSJ} / 4$ precipitating particle measurements taken poleward of $50^{\circ}$ north magnetic latitude by the DMSP F8 satellite during November and December 1989 and January 1990 and by the F9 satellite during December 1989 and January 1990; the solar minimum data base includes F6 and F7 satellite measurements 
taken in the same region during December 1986 and January 1987. Also, SSJ/4 measurements taken by the F8 satellite during January 1988 represent solar medium. Select SSIES ambient plasma density data are included for the F8 and F9. A summary of the data base including the number of satellite orbits is given in Table 1 . Winter months were chosen because they coincide with extended periods of eclipsed flight (minimizes photoemission) and were believed to provide highlatitude ionospheric conditions more conducive to spacecraft charging (lower ambient plasma densities). The $10.7-\mathrm{cm}$ solar fluxes and 3-hour $K_{p}$ sums during these periods are shown in Figure 2. $K_{p}$ values show variability throughout the periods, but the average $K_{p}$ is highest at solar maximum and lowest at solar minimum. The solar maximum and minimum data periods have average $F_{10.7}$ values of 213 and 70 , respectively, which are very representative of such conditions. The third data period which we define to be solar medium has an average $F_{10.7}$ value of only 105 which is somewhat lower than normally used to define medium solar activity (i.e., $F_{10.7}$ of $\sim 130$ ). During the solar maximum and minimum periods the satellites reported SSJ/4 data well over $90 \%$ of the time (no down days) and generated over 5 million electron/ion spectra during nearly 4000 northern hemisphere passes; the month of solar medium data is less complete with six down days and includes measurements taken during 275 orbits. Satellite-to-plasma potential differences were identified using the SSJ/4 ion spectra.

When a spacecraft charges negatively, the ambient positive ions are accelerated to the spacecraft and gain an energy equal to $-q \phi$, where $q$ is the ions' charge and $\phi$ is the satellite potential. The resulting ion differential number flux depicts an intense flux of ions with energies equal to $-q \phi$ with negligible fluxes at lower energies, and the reported satellite charging level (-volts) is defined as the central energy of the SSJ/4 channel measuring the enhanced ion flux. This method of identifying satellite-to-plasma potential differences has been used by DeForest [1972], Mullen and Gussenhoven [1982], and Gussenhoven et al. [1985] and is the principle technique used in this study.

Using the electrostatic principle just described, a computer code examined over 5.3 million ion spectra for a charging signature. Because ion precipitation of magnetospheric origin possesses multiple distributions [Hardy et al., 1989], care was taken not to label a precipitating ion spectrum as representing an acceleration of the local ion population. The code was general enough to select all charging-like signatures, and then each of these spectra was examined in detail. Though ion precipitation in a narrow energy band often resembles a charging signature at a given moment, the ion spectra before and after the spectrum in question reflects the nature of the environment.

As a satellite orbits through a region of ion precipitation, measured ion energies and number fluxes are likely to be ordered from one second to the next though they may change rapidly. In contrast, the ions' energies and number fluxes associated with a charging event are more unordered (random) as charging levels constantly fluctuate and the SSJ/4 sweeps through one energy channel every tenth of a second. Also, the presence of energetic electron precipitation is necessary to generate significant negative potentials. Thus the presence or

TABLE 1. Extent of the Charging Data Base

\begin{tabular}{|c|c|c|c|c|c|}
\hline Solar Activity & Year & November & December & January & $\begin{array}{c}\text { Total Number of } \\
\text { Orbits }\end{array}$ \\
\hline $\begin{array}{l}\text { Minimum } \\
\text { Minimum } \\
\text { Medium } \\
\text { Maximum } \\
\text { Maximum }\end{array}$ & $\begin{array}{l}1986 \\
1987 \\
1988 \\
1989 \\
1990\end{array}$ & 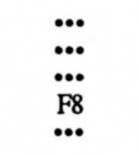 & 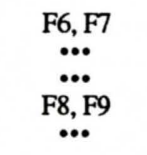 & 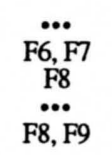 & $\begin{array}{c}840 \\
865 \\
275 \\
1285 \\
795\end{array}$ \\
\hline
\end{tabular}

SSIES data is included for the F8 and F9 satellites.
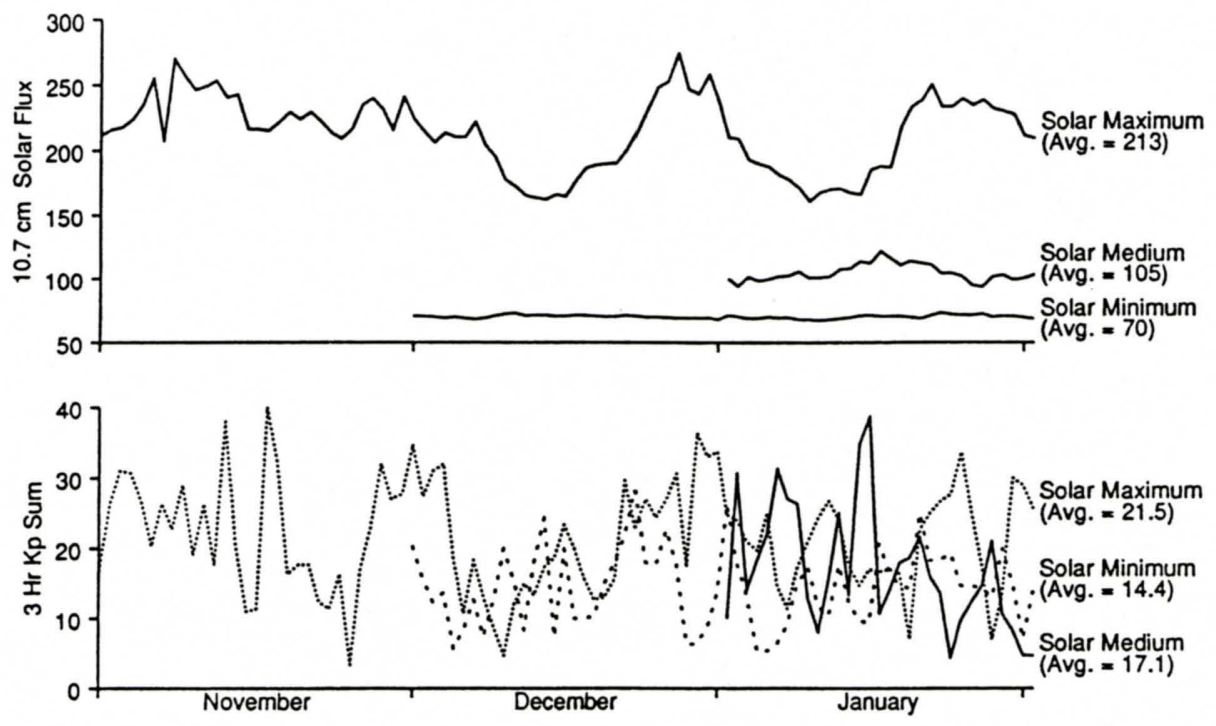

Fig. 2. (Top panel) Solar flux levels and (lower panel) 3-hourly $K_{p}$ values for the times spanned by the charging data base. 
absence of high-energy electron precipitation further aids the identification process though the SSJ/4's inability to measure particles above $30 \mathrm{keV}$ must be considered. The result of collectively using these selection tools was to discount several ion precipitation spectra and SSJ/4 anomalous readings as representing genuine satellite charging. In addition to the search for charging events via SSJ/4 ion measurements, the analysis included examining the environments during periods of energetic electron precipitation for which charging did not occur.

On the basis of the electron fluxes during the DMSP charging events reported by Gussenhoven et al. [1985], all periods for which the electron fluxes $\geq 9.6 \mathrm{keV}$ exceeded $3 \mathrm{x}$ $10^{8}$ electrons $/\left(\mathrm{cm}^{2} \mathrm{sec}\right.$ ster) were quantified to characterize the frequency of energetic electron precipitation known to generate charging. These values are used to compare the precipitating electron environments of solar maximum, medium, and minimum. The thermal plasma densities are considered (F8 and F9 only) for these periods of enhanced energetic electron fluxes and for charging events. In this way, the environments which generate and inhibit charging are better described.

\section{DMSP CHARGING RESULTS}

\subsection{Solar Minimum}

Spacecraft-to-plasma negative potential differences occurred often during solar minimum conditions. The DMSP satellites charged negatively on 169 occasions with peak potentials ranging from $-47 \mathrm{~V}$ to $-1.4 \mathrm{kV}$; a summary of the events' magnitudes and durations are shown in Figure 3. The charging levels shown represent the greatest potential achieved during the entire event and the durations shown represent a presence of a negative charging level $\geq 47 \mathrm{~V}$. The large number of events precludes a detailed presentation of each, and while each charging case is unique in some manner, many of the events are similar in location.

The locations of the 169 solar minimum charging events are shown in Figure $4 a$. Nearly $85 \%$ of the events occurred between $68^{\circ} \mathrm{N}$ and $75^{\circ} \mathrm{N}$ magnetic latitude and between 2100 and 0100 magnetic local time. The events are sorted by satellite and are depicted in Figures $4 b$ (F6) and $4 c$ (F7) which show that the lack of events identified in the post-midnight sector is not due to limited orbital coverage. All charging events occurred either within full shadow of the Earth or occasionally $(<5 \%)$ just along the eclipse border such that the SSJ/4 remained in shadow. The dependence on magnetic location and darkness also yields a UT dependence as nearly $70 \%$ of the events occurred between 0630 and 1830 UT. The durations of these events range from $1 \mathrm{sec}$ to $1 \mathrm{~min}$ (see Figure 3 ) and illustrate the variability of the charging currents, and the high energy precipitating electrons are a charging current source of particular interest.

Energetic electrons are accepted as a primary cause of significant negative spacecraft charging. To characterize the electron population during the charging events of solar minimum, Figure 5 depicts plots of total ( $30 \mathrm{eV}$ to $30 \mathrm{keV}$ ) electron integral number flux (top panel) and $\geq 9.6 \mathrm{keV}$ electron integral number flux (lower panel) versus charging magnitude. As Gussenhoven et al. [1985] found, the correlation of total electron flux to charging is weak, and these fluxes range over 1 order of magnitude for a given potential. Periods of $\geq 9.6 \mathrm{keV}$ electrons fluxes exceeding $3 \times 10^{8}$ electrons $/\left(\mathrm{cm}^{2} \mathrm{sec}\right.$ ster $)$ in darkness for more than $2 \mathrm{sec}$ resulted in negative charging $(>46 \mathrm{~V})$ more than $50 \%$ of the time. The $\geq 9.6 \mathrm{keV}$ plot depicts a better correlation of electron fluxes to potential, but the fluxes extend over a large range particularly at lower charging levels. The unorderedness and broad range of this latter plot are due to four factors which are not taken into account; first, the ambient plasma densities probably vary for different events; second, the electron population below $1 \mathrm{keV}$ may create more than one secondary electron for each incident electron and inhibit charging [Katz et al., 1977; Leung et al., 1981]; third, electrons from 2 to 9 $\mathrm{keV}$ may contribute to charging; and finally, electron fluxes beyond the SSJ/4's energy range may be contributing to the charge imbalance. While the better correlation includes the $\geq 9.6 \mathrm{keV}$ electrons, many of the events demonstrate that lesser energetic electrons can drive DMSP charging.

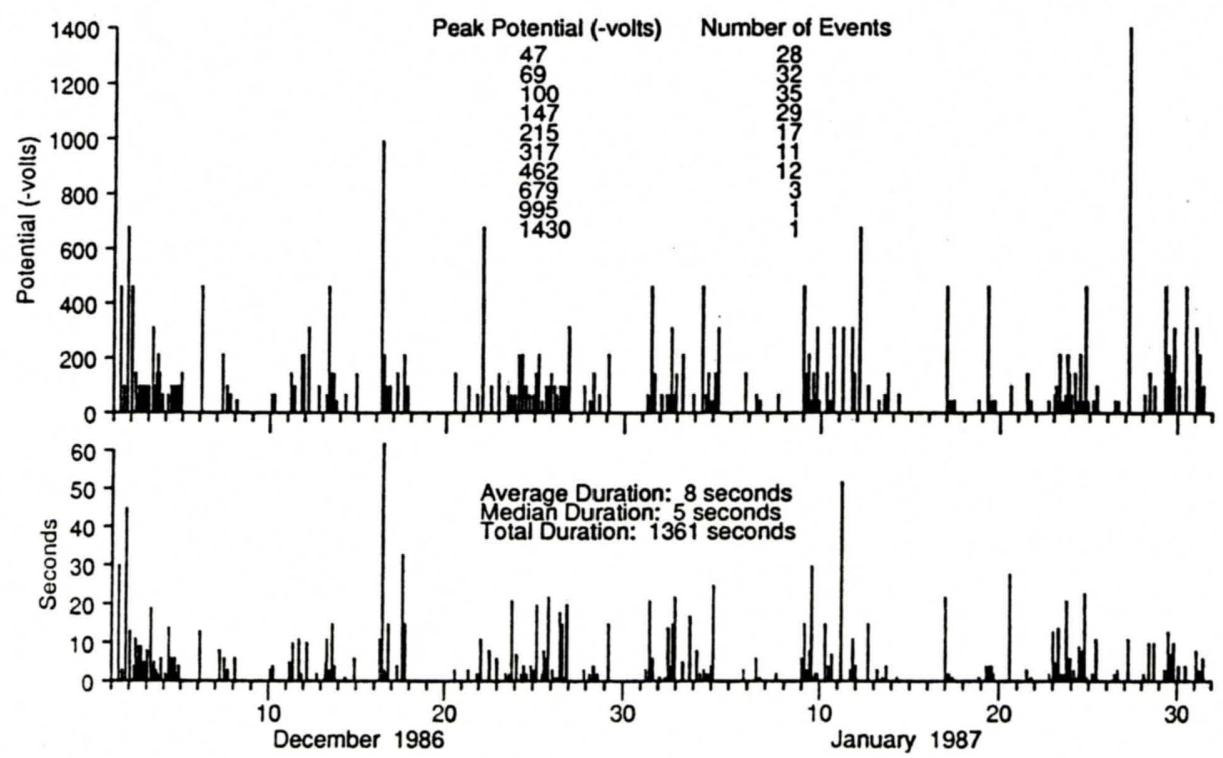

Fig. 3. (Top panel) Maximum charging potentials and (lower panel) charging durations for the DMSP F6 and F7 satellites during solar minimum. 
a

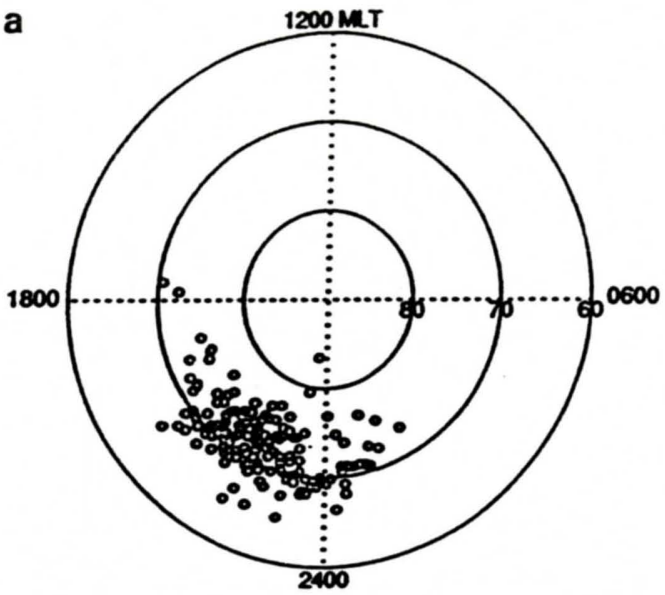

b

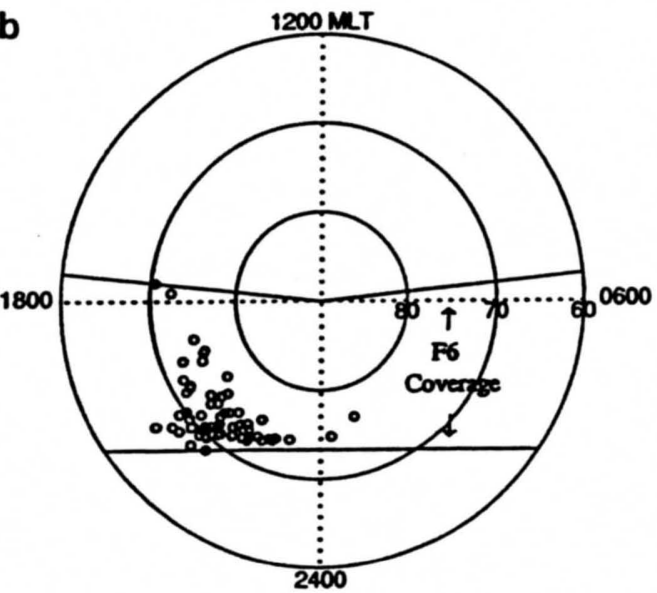

c

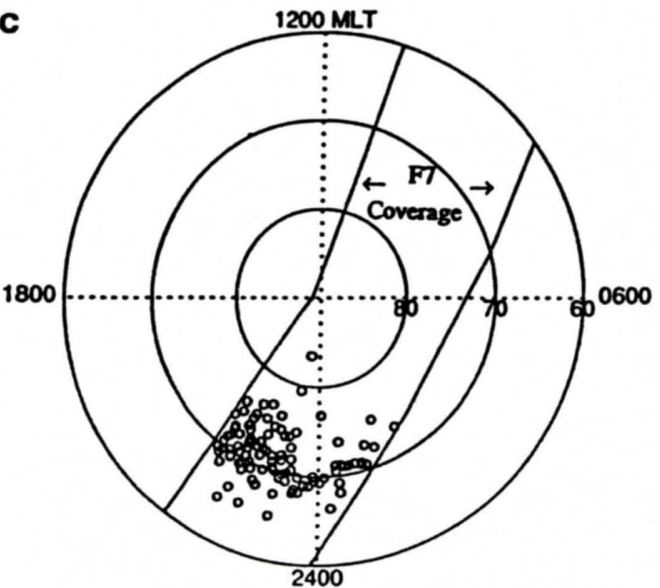

Fig. 4. Locations of the solar minimum charging events in a corrected geomagnetic latitude and magnetic local time polar plot for the (a) combined F6 and F7 satellite, (b) F6 satellite, and (c) F7 satellite. Each circle represents the location of the greatest charging potential.

Though the study of Gussenhoven et al. [1985] showed a direct relationship between DMSP charging and enhanced fluxes of electrons with energies from 14 to $30 \mathrm{keV}$, electrons of lesser energy produced significant charge imbalances during solar minimum. Charging levels as high as $-100 \mathrm{~V}$ were achie:"ed with electron fluxes $\leq 6.5 \mathrm{keV}$, and electron populations during several events below $100 \mathrm{~V}$ suggest that 2 to $4.5 \mathrm{keV}$ electrons can drive charging. Though other current sources such as ambient plasma may enhance the probability or severity of charging, only precipitating electrons appear
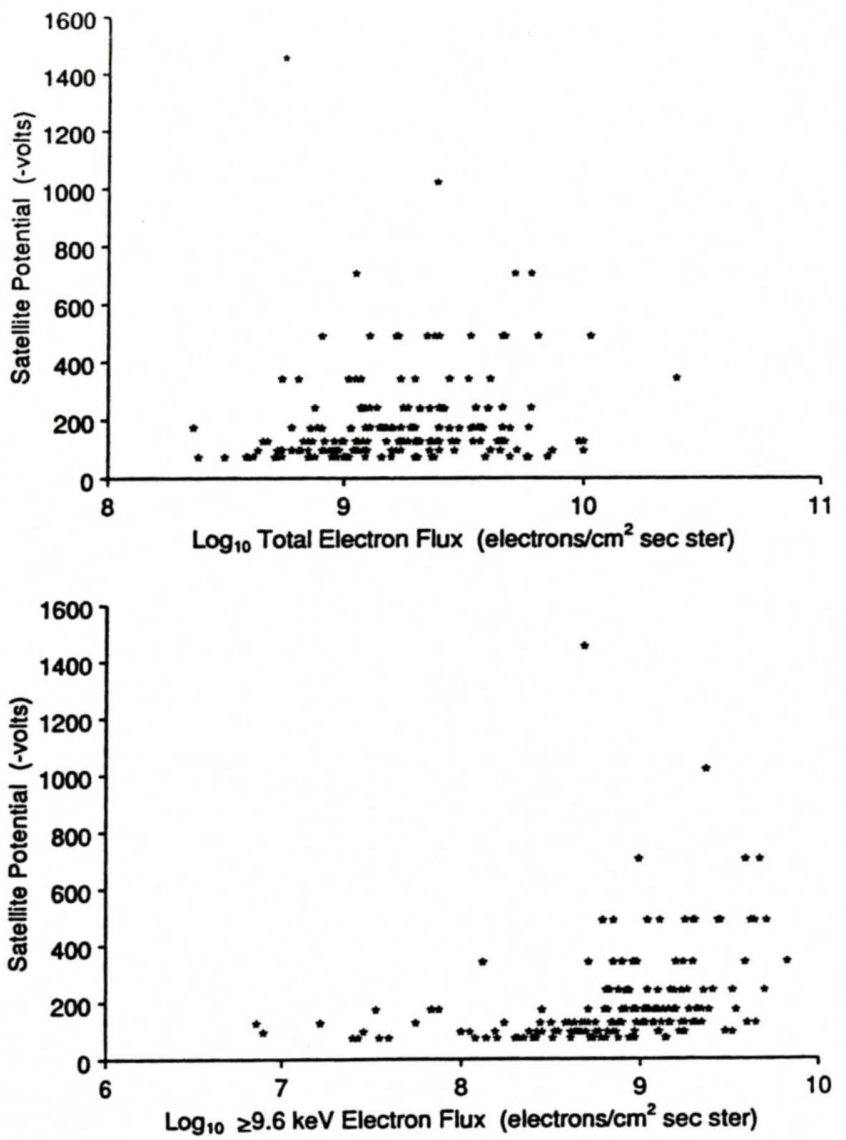

Fig. 5. Satellite potential as a function of the (top panel) total electron flux and the (lower panel) $\geq 9.6 \mathrm{keV}$ electron flux.

capable of generating these negative potentials. Considering that 2 to $6.5 \mathrm{keV}$ electrons likely contribute to charging and that incident electrons at lower energies limit charging, a further analysis of the electron populations during the charging events is necessary.

The ratio of the $\geq 3 \mathrm{keV}$ electron flux to the total electron flux during charging illustrates the effect of enhanced secondary electron production due to incident electrons $\leq 1$ $\mathrm{keV}$. Table 2 lists three groups of events based on charging level. Group A corresponds to negative charging potentials peaking between 45 and $69 \mathrm{~V}$; group B represents potentials between 100 and $215 \mathrm{~V}$; and group $\mathrm{C}$ includes charging levels

TABLE 2. Electron Spectra Characteristics During Solar Minimum Charging Events

Charging level, $-\mathrm{V}$

\begin{tabular}{|c|c|c|c|}
\hline & $45-69^{a}$ & $100-215^{b}$ & $\geq 314^{c}$ \\
\hline Number of events & 60 & 81 & 28 \\
\hline $\begin{array}{l}\% \text { of events with } \\
\geq 3 \mathrm{keV} \text { fluxes } \\
>10^{9} \text { electrons/ } \\
\mathrm{cm}^{2} \text { sec ster }\end{array}$ & $32 \%$ & $65 \%$ & $64 \%$ \\
\hline $\begin{array}{l}\% \text { of events with } \\
\geq 3 \mathrm{keV} \text { flux/total } \\
\text { flux } \geq 90 \%\end{array}$ & $38 \%$ & $50 \%$ & $82 \%$ \\
\hline
\end{tabular}

${ }^{a}$ Data are for group $\mathrm{A}$

${ }^{b}$ Data are for group B.

${ }^{c}$ Data are for group $\mathrm{C}$. 
greater than $314 \mathrm{~V}$. Of particular interest is a comparison of group $\mathrm{B}$ to group $\mathrm{C}$. The percent of events having large fluxes of $\geq 3 \mathrm{keV}$ electrons is the same for groups B and C, but the electron populations for over $80 \%$ of the group $C$ events are $90 \%$ energetic. This illustrates that group $\mathrm{C}$ events have fewer incident electrons $\leq 1 \mathrm{keV}$, and therefore fewer secondary electrons are generated. The lower DMSP charging levels of group A include not only decreased $\geq 3 \mathrm{keV}$ fluxes but also higher percentages of lower energy electrons.

Two severe charging events during solar minimum exemplify the process of large energetic electron fluxes combining with small fluxes of lower-energy electrons (group $\mathrm{C}$ events) to generate significant potentials. The $-1.43 \mathrm{kV}$ and $-995 \mathrm{~V}$ charging events on January 27,1987 , and December 16,1986 , respectively, represent the two largest DMSP negative potentials reported to date. Both occurred near $70^{\circ} \mathrm{N}$ magnetic latitude and at 2215 MLT. The precipitating electron spectra during both events are similar in that the greatest number flux is due to electrons $>20 \mathrm{keV}$, and both spectra suggest that large electron fluxes exist beyond $30 \mathrm{keV}$. A plot of the electron fluxes above $1 \mathrm{keV}$ for both events is shown in Figure 6. In particular, the $-1.43 \mathrm{kV}$ event has relatively small electron fluxes below $20 \mathrm{keV}$ and shows a logarithmically steady flux increase from 10 to $30 \mathrm{keV}$. The $-1.43 \mathrm{kV}$ potential of January 27 was achieved for only $1 \mathrm{sec}$, while a 1 $\mathrm{kV}$ potential was reached twice during the event on December 16. Unfortunately, the detectors' ranges do not permit a more adequate description of the electron spectra driving these extreme potentials.

The total number of seconds that the F6 and F7 satellites encountered intense energetic electron precipitation is shown in Table $3 a$, and the total time that the satellites encountered intense electron precipitation of lesser energy is shown in Table $3 b$. Values in parentheses represent the percentage of the total time that the satellites encountered these fluxes poleward of $50^{\circ} \mathrm{N}$ magnetic latitude. Statistically, the two

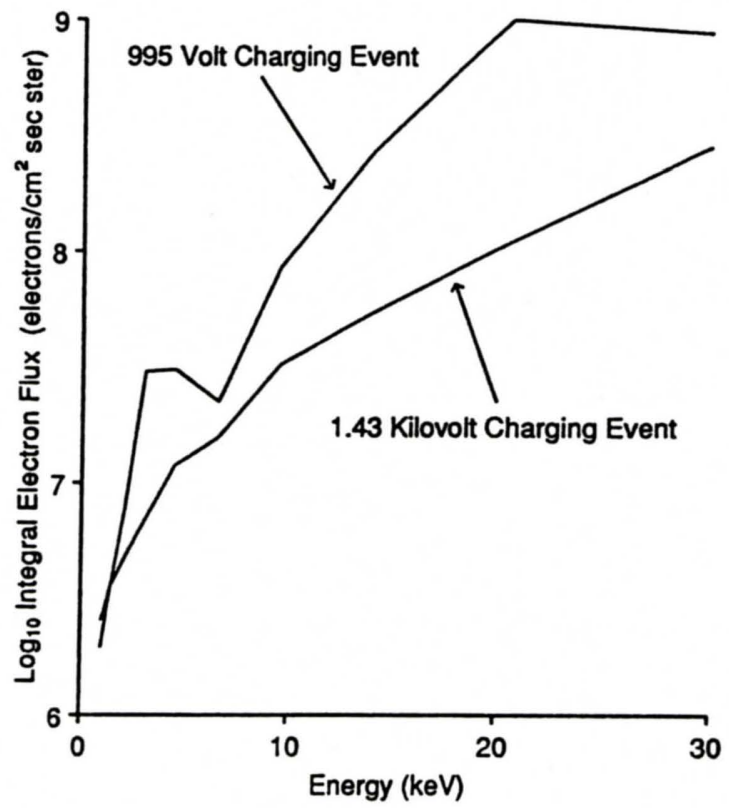

Fig. 6. Electron number flux spectra for the two most severe charging events. The $-995 \mathrm{~V}$ potential occurred on December 16, 1986, at $1000: 48 \mathrm{UT}$, and the $-1.4 \mathrm{kV}$ potential occurred on January 27,1987 , at 0412:38 UT.
TABLE 3a. Total Time of $29.6 \mathrm{keV}$ Electron Fluxes Greater Than $3 \times 10^{8}$ electrons $/ \mathrm{cm}^{2} \mathrm{sec}$ ster During Solar Minimum

\begin{tabular}{lcc}
\hline & \multicolumn{2}{c}{ Satellite } \\
\cline { 2 - 3 } & \multicolumn{2}{c}{ F6 } \\
\hline December 1986 & $773(0.14 \%)$ & $502(0.09 \%)$ \\
January 1987 & $370(0.06 \%)$ & $528(0.09 \%)$ \\
\hline
\end{tabular}

Values in parentheses represent the percentage of the total time that the satellites encountered these fluxes poleward of $50^{\circ} \mathrm{N}$ magnetic latitude. Time is in seconds.

TABLE $3 b$. Total Time of Total Electron Fluxes Greater Than $2 \times 10^{9}$ electrons $/ \mathrm{cm}^{2} \mathrm{sec}$ ster During Solar Minimum

\begin{tabular}{|c|c|c|}
\hline & \multicolumn{2}{|c|}{ Satellite } \\
\hline & F6 & F7 \\
\hline December 1986 & $3039(0.55 \%)$ & $4017(0.72 \%)$ \\
\hline January 1987 & $2185(0.37 \%)$ & $3855(0.66 \%)$ \\
\hline
\end{tabular}

Values in parentheses represent the percentage of the total time that the satellites encountered these fluxes poleward of $50^{\circ} \mathrm{N}$ magnetic latitude. Time is in seconds.

satellites have a similar occurrence frequency $\sim 0.1 \%$ of the time for Table $3 a$ and $\sim 0.6 \%$ for Table $3 b$, the latter being slightly assymmetric with the F7 satellite having the larger occurrence frequency. High-energy, high-flux values are significantly less frequent than enhanced fluxes spanning the entire energy spectrum.

\subsection{Solar Medium}

Satellite charging occurred 14 times during the 25 days of solar medium and ranged from -45 to $-310 \mathrm{~V}$. A summary of the events' magnitudes and durations is shown in Figure 7. Though the data base is considerably smaller than that of solar minimum, the charging results are similar.

The events occurred in darkness, and as shown in Figure 8, most charging occurred in the magnetic pre-midnight sector and near $70^{\circ} \mathrm{N}$ magnetic latitude. However, this distribution of charging locations shows four events in the morning sector in a region devoid of charging during solar minimum (compare Figures $4 a$ and 8). This difference is discussed briefly in section 5. Measured ambient plasma densities (SSIES) for these events range from $8 \times 10^{1}$ to $9 \times 10^{3} \mathrm{~cm}^{-3}$. As observed in some of the solar minimum events, charging up to $-144 \mathrm{~V}$ appears to be the result of electron fluxes $\leq 4.5 \mathrm{keV}$. Total electron fluxes extend from $5 \times 10^{8}$ to $1.6 \times 10^{10}$ electrons/ $\left(\mathrm{cm}^{2} \mathrm{sec}\right.$ ster), while $\geq 9.6 \mathrm{keV}$ fluxes measure from $1 \times 10^{6}$ to 4 $\times 10^{9}$ electrons $/\left(\mathrm{cm}^{2} \mathrm{sec}\right.$ ster $)$, each showing a broad range similar to the solar minimum events. The frequency that the F8 satellite encountered enhanced electron fluxes is shown in Table 4. To compare these values to those of solar minimum, a normalized frequency for solar medium is calculated because of the smaller data base of January 1988. The normalized frequency of energetic precipitation $(\geq 9.6 \mathrm{keV}$ electron number flux) is greatest during solar medium, but the normalized number of F8 solar medium charging events is still below the number of F6 solar minimum events. This, of course, is a crude comparison, but these results suggest that the environment 

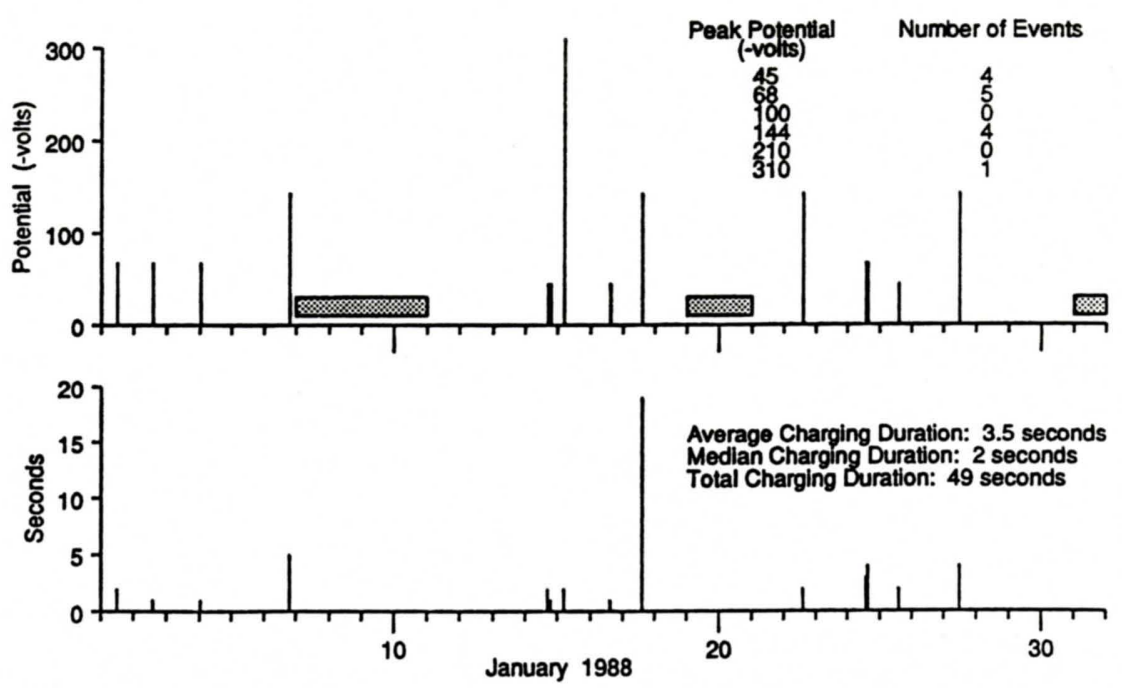

Fig. 7. (Top panel) Maximum charging potentials and (lower panel) charging durations for the DMSP F8 satellite during solar medium. Shading represents periods for which SSJ/4 data was not available.

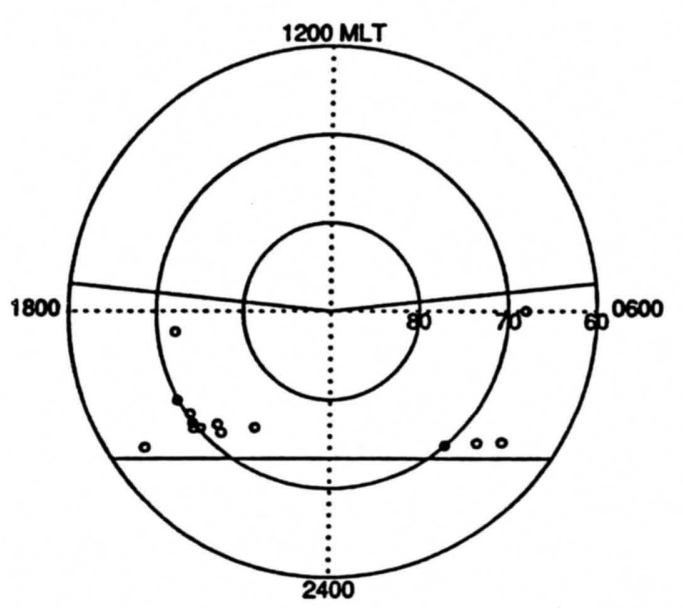

Fig. 8. Locations of the solar medium charging events in a corrected geomagnetic latitude and magnetic local time polar plot for the F8 solar medium charging events.

during solar minimum is more conducive to spacecraft charging.

\subsection{Solar Maximum}

Spacecraft charging as observed during solar minimum and solar medium was absent during solar maximum conditions. A DMSP satellite charged to $-46 \mathrm{~V}$ only one time, and this relatively weak potential lasted just 2 sec. The environments which generated this single charging event and inhibited others from occurring are examined by analyzing the electron spectra, quantifying the energetic electron precipitation, and identifying the measured ambient plasma densities within regions of energetic electron precipitation.

The charging event during solar maximum is summarized in Table 5. Nearly half of the total precipitating electron integral number flux is due to electrons $\geq 3 \mathrm{keV}$, while electrons $\geq 9.6$ $\mathrm{keV}$ contribute over $17 \%$ toward total number flux. This electron distribution represents the fifth largest number flux of $\geq 3 \mathrm{keV}$ electrons in the entire solar maximum data base. The thermal plasma density of $2.0 \times 10^{4} \mathrm{~cm}^{-3}$, though greater than all of the densities during the solar medium charging events and those reported by Gussenhoven et al. [1985], represents one of the lower plasma densities identified in this study during solar maximum periods of enhanced energetic electron precipitation. Only on two other occasions were the electron fluxes more energetic and the thermal plasma density less dense, and detectable charging did not occur. In both instances, not only were electron fluxes above $1 \mathrm{keV}$ greater than those during the charging event, but electron fluxes below $1 \mathrm{keV}$ were likewise significantly greater than electron fluxes below $1 \mathrm{keV}$ during the charging event, so the increased secondary electron production may explain the absence of charging during these two cases.

The total number of seconds that the F8 and F9 satellites encountered intense energetic electron precipitation is shown in Table $6 a$. Listed in Table $6 b$ is the total time that the satellites encountered intense electron precipitation of lesser energy. These values characterize the occurrence of large electron fluxes and may be compared to those during solar minimum and medium given in Tables $3 a, 3 b$, and 4 . The percentage of total time that the $\geq 9.6 \mathrm{keV}$ electron fluxes exceeded $3 \times 10^{8}$ electrons $/\left(\mathrm{cm}^{2} \mathrm{sec}\right.$ ster $)$ is almost identical during solar minimum and solar maximum. In summary, on the basis of the electron distributions during the charging events of solar minimum and medium and of those reported by Gussenhoven et al. [1985], the electron fluxes during solar maximum were energetic enough and encountered often enough to generate numerous charging events. Since charging did not occur, the ambient plasma densities which accompanied the energetic electron precipitation are examined.

Measured thermal plasma densities (SSIES) ranged from 1.3 $\times 10^{4}$ to $1.3 \times 10^{5} \mathrm{~cm}^{-3}$ (with two exceptions) during periods of enhanced electron precipitation within the Earth's shadow. Precipitating electron distributions during several of these periods resemble those of the solar minimum, medium, and Gussenhoven et al. [1985] for which charging did occur. In comparing specific cases, only the thermal plasma density varies significantly between the environments; the measured densities are 1 to 3 orders of magnitude greater during solar maximum.

\section{Discussion}

One hundred eighty-four DMSP charging events were identified by an extensive search of F6, F7, F8, and F9 
TABLE 4. Total Time of Electron Integral Number Fluxes [electrons $/\left(\mathrm{cm}^{2} \mathrm{sec}\right.$ ster $)$ ] During Solar Medium Compared to Solar Minimum

\begin{tabular}{lccc}
\hline \multicolumn{4}{c}{ During Solar Medium Compared to Solar Minimum } \\
\hline & $\begin{array}{c}\geq 9.6 \mathrm{keV} \\
\text { Electron } \\
\text { Flux } \geq 3 \times 10^{8}\end{array}$ & $\begin{array}{c}\text { Total } \\
\text { Electron } \\
\text { Flux } \geq 2 \times 10^{9}\end{array}$ & $\begin{array}{c}\text { Number } \\
\text { of Events }\end{array}$ \\
\hline Solar medium, Jan. 1988 (F8) & 307 & 1037 & 14 \\
Normalized, Jan. 1988 (F8) & 460 & 1550 & 21 \\
Solar minimum, Jan. 1987 (F6) & 370 & 2185 & 27 \\
\hline
\end{tabular}

Time is in seconds.

TABLE 5. Summary of the Single Charging Event During Solar Maximum

\begin{tabular}{ll}
\hline \multicolumn{1}{c}{ Value } & \multicolumn{1}{c}{ Characteristic } \\
\hline Date/Time & November 13, 1989; 2231:50 UT \\
Satellite & $\mathrm{F}$ \\
Magnetic latitude & $67^{\circ} \mathrm{N} *$ \\
Magnetic local time & $0450^{*}$ \\
Peak potential & $-45 \mathrm{~V}$ \\
Total electron integral flux & $2.7 \times 10^{10}$ electrons $/ \mathrm{cm}^{2} \mathrm{sec}$ ster \\
$\geq 3.0 \mathrm{keV}$ electron integral flux & $1.2 \times 10^{10}$ electrons $/ \mathrm{cm}^{2} \mathrm{sec}$ ster \\
$\geq 9.7 \mathrm{keV}$ electron integral flux & $4.8 \times 10^{9}$ electrons $/ \mathrm{cm}^{2} \mathrm{sec} \mathrm{ster}$ \\
Ambient plasma density & $2.0 \times 10^{4} \mathrm{~cm}^{-3}$ \\
\hline
\end{tabular}

* The satellite is just out of eclipse though the SSJ/4 remains in shadow.

TABLE 6a. Total Time of $\geq 9.6 \mathrm{keV}$ Electron Fluxes Greater Than $3 \times 10^{8}$ electrons $/ \mathrm{cm}^{2} \mathrm{sec}$ ster During Solar Maximum

\begin{tabular}{lcc}
\hline & \multicolumn{2}{c}{ Satellite } \\
\cline { 2 - 3 } & F8 & P9 \\
\hline November 1989 & $410(0.07 \%)$ & $\ldots$ \\
December 1989 & $530(0.09 \%)$ & $703(0.12 \%)$ \\
January 1990 & $415(0.08 \%)$ & $534(0.10 \%)$ \\
\hline
\end{tabular}

Values in parentheses represent the percentage of the total time that the satellites encountered these fluxes poleward of $50^{\circ} \mathrm{N}$ magnetic latitude. Time is in seconds.

TABLE $6 b$. Total Time of Total Electron Fluxes Greater Than $2 \times 10^{9}$ electrons $/ \mathrm{cm}^{2} \mathrm{sec}$ ster During Solar Maximum

\begin{tabular}{|c|c|c|}
\hline & \multicolumn{2}{|c|}{ Satellite } \\
\hline & $\mathrm{FB}$ & $\mathrm{P9}$ \\
\hline November 1989 & $1306(0.23 \%)$ & $\bullet$ \\
\hline December 1989 & $1745(0.30 \%)$ & $3204(0.56 \%)$ \\
\hline January 1990 & $1485(0.28 \%)$ & $2753(0.51 \%)$ \\
\hline
\end{tabular}

Values in parentheses represent the percentage of the total time that the satellites encountered these fluxes poleward of $50^{\circ} \mathrm{N}$ magnetic latitude. Time is in seconds.

measurements taken during winter months of solar minimum, medium, and maximum; 169 of the events, including the most severe DMSP potential ever reported $(-1.4 \mathrm{kV})$, occurred during solar minimum while only one event occurred during solar maximum. However, not only are the number of events different, their duration and potential history are different (see Figures 3 and 7). We have combined these three quantities on a daily basis into a single parameter we call the charging power. This parameter is then useful for relative comparisons between the three periods. Figure 9 depicts a daily "charging power" for all three periods and illustrates the observed solar cycle dependence. The charging power is defined daily as the integral of the acceleration potential with respect to time. The primary current sources which can generate or inhibit these DMSP charging levels are photoemission, precipitating ions, precipitating electrons and their secondary electrons, and ambient ions [Garrett, 1981; Yeh and Gussenhoven, 1987]. Thus a significant variation during the solar cycle of one or more of these current sources is believed to be responsible for the extreme contrast in results.

Since nearly all charging occurred in full shadow of the Earth, and the amount of time the satellites spent in eclipse during the three periods was chosen to be similar, a varying photoemission rate could not have caused the resulting solar cycle dependence. Enhanced ion precipitation occasionally contributed toward current balance by occurring simultaneously with energetic electron precipitation, but the frequency of this phenomenon was insignificant. Furthermore, simultaneous electron/ion precipitation did not occur more often during solar maximum than during solar minimum, so a variation of ion precipitation did not contribute to the charging solar cycle dependence. The frequency and distribution of energetic electron precipitation did not vary significantly between the periods of study (see Tables 3, 4, and 6), and electron fluxes which generated charging during solar minimum did not cause charging during solar maximum.

The ambient plasma is the remaining current balance term. Measured ion densities during periods of enhanced electron precipitation were greater during solar maximum than solar medium, and it is likely that thermal plasma densities were even lower during solar minimum. A model is now introduced to characterize the thermal plasma at $840 \mathrm{~km}$ during solar minimum and to identify the theoretical plasma density ranges over a solar cycle.

The time-dependent ionospheric model (TDIM) is a global scale, three-dimensional, and time-dependent computer model which numerically solves the continuity, momentum, and energy equations for ions and the energy equation for electrons 


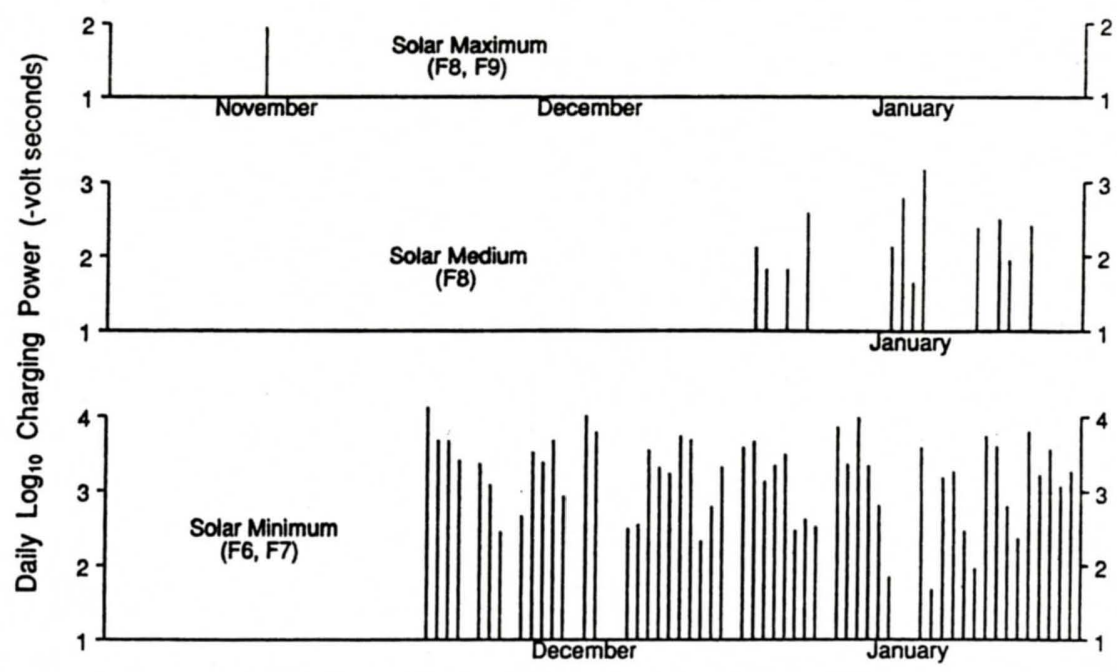

Fig. 9. Comparison of the daily charging power for solar maximum, medium, and minimum. The charging power for a charging event is the integral of the charging potential with time.

between 120 and $800 \mathrm{~km}$. A detailed description of the TDIM and several model-to-observation comparisons are given in a review by Sojka [1989], and a mathematical presentation of the TDIM's methodology is given by Schunk [1988]. Though the DMSP spacecraft orbit nearly $40 \mathrm{~km}$ above the model's upper boundary, significant ambient plasma density gradients between 800 and $840 \mathrm{~km}$ are not normally present because of the large plasma scale heights at these altitudes. Thus TDIM output for $800 \mathrm{~km}$ predicts the ambient plasma densities encountered by the satellites and provides key input in analyzing the results of solar minimum charging.

To illustrate the high-latitude plasma variation predicted by the TDIM, the plasma densities at $800 \mathrm{~km}$ and 1300 UT for solar minimum, winter, low $K_{p}$, and interplanetary magnetic field $B_{y}$ positive conditions are shown in Figure 10. Arrows identify the regions of most interest for this study. Region A represents the polar hole, a region of significantly depleted

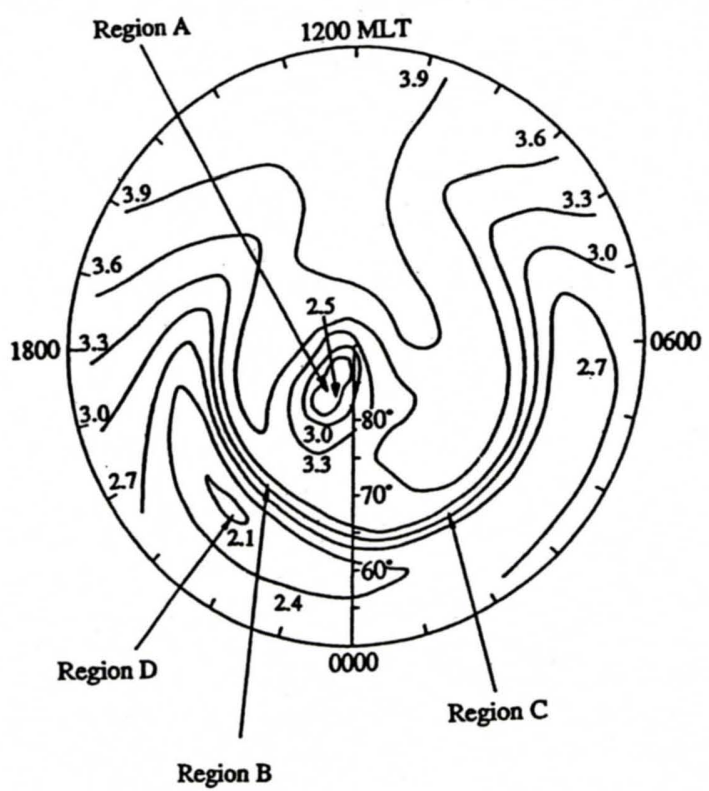

Fig. 10. Contours of the TDIM electron density at $800 \mathrm{~km}$ in a corrected geomagnetic latitude and magnetic local time polar plot. The densities are for winter, solar minimum, and low magnetic activity conditions. Contours are of the logarithm base 10 of the electron density $\left(\mathrm{cm}^{-3}\right)$. plasma. Regions B and C denote pre-midnight and postmidnight regions of aurorally enhanced plasma density. Further equatorward, region $\mathrm{D}$ depicts the mid-latitude trough. The general morphology shown in Figure 10 does not change significantly for varying conditions of $K_{p}$, universal time, and solar flux. However, the location of the polar hole for $B_{y}$ negative conditions is centered near 0200 MLT rather than near 2200 MLT. The modeled densities within the key regions may be compared for various conditions.

TDIM plasma densities for solar minimum, medium, and maximum, and low and medium $K_{p}$ activities are shown in Table 7. Densities are lowest in all regions during solar minimum and range over 1 order of magnitude or more from solar minimum to solar maximum within the polar hole and mid-latitude trough. Densities in the aurorally enhanced regions during solar maximum are 3.5 to 4.5 times greater than during solar minimum, and in general, the model densities for solar maximum and medium are consistent with the SSIES data reported in section 4 . While a large variation over a solar cycle is predicted, the densities during solar minimum are of special interest.

Considering the location of most of the charging events, the area of primary interest includes the transition between the polar hole and pre-midnight aurorally enhanced region. The location of the polar hole's equatorward boundary is due to a statistical model input of auroral precipitation at high magnetic latitudes which limits the equatorward extension of the hole. If this statistical auroral precipitation was replaced with realistic discrete precipitation, the polar hole flux tubes would convect further equatorward before encountering the first auroral structure. This would lead to significantly more depleted flux tubes in the vicinity of the charging events than indicated by the densities in Table 7 .

In summary, the TDIM predicts at least an order of magnitude variation in ambient plasma density at high magnetic latitudes between solar maximum and minimum, and the model data closely represents the measured data of solar maximum and medium as well as the experimental data of Gussenhoven et al. [1985]. From these model outputs and experimental results an ambient plasma density of $10^{4} \mathrm{~cm}^{-3}$ may be considered as a rough threshold for DMSP charging assuming precipitating electron distributions are similar to those of this study and of Gussenhoven et al. [1985]. The TDIM predicts densities below 
TABLE 7. Model Ambient Plasma Densities $\left(\log _{10} \mathrm{~cm}^{-3}\right)$ at $800 \mathrm{~km}$ and 1300 UT for Three Levels of Solar Flux and Low and Medium Levels of Magnetic Activity

$10.7 \mathrm{~cm}$ Solar Flux

70

130

210

\begin{tabular}{lccc} 
Region A (polar hole) & $2.48 / 2.80$ & $2.90 / 3.40$ & $3.40 / 3.80$ \\
$\begin{array}{l}\text { Region B (aurorally } \\
\text { enhanced 2200 MLT) }\end{array}$ & $3.72 / 3.95$ & $4.05 / 4.30$ & $4.35 / 4.55$ \\
$\begin{array}{l}\text { Region C (aurorally } \\
\text { enhanced 0200 MLT) }\end{array}$ & $3.70 / 3.70$ & $3.90 / 4.10$ & $4.25 / 4.25$ \\
$\begin{array}{l}\text { Region D (mid-latitude } \\
\text { trough) }\end{array}$ & $1.91 / 2.25$ & $2.54 / 3.00$ & $3.21 / 3.50$ \\
\hline
\end{tabular}

The $K p$ level is $1 / 3.5$.

$10^{4} \mathrm{~cm}^{-3}$ during solar minimum and above $10^{4} \mathrm{~cm}^{-3}$ during solar maximum for much of the nightside at $800 \mathrm{~km}$. The measured plasma density during the weak charging event of solar maximum was just above $10^{4} \mathrm{~cm}^{-3}$, while densities for 10 out of the 11 charging events reported by Gussenhoven et al. [1985] were below $10^{4} \mathrm{~cm}^{-3}$, and densities during all of the solar medium events were below $10^{4} \mathrm{~cm}^{-3}$. The electron fluxes causing charging during solar minimum did not cause charging during solar maximum when measured ambient plasma densities were above $10^{4} \mathrm{~cm}^{-3}$. Hence, the solar cycle control of the charging is due to the solar cycle control of the ambient plasma.

Many studies emphasize that a flux of "energetic" electrons is necessary in order to generate significant negative potentials. Though DMSP charging levels during solar minimum and medium correlated better to fluxes of highenergy electrons $(\geq 9.6 \mathrm{keV})$ than to total electron fluxes, potentials as large as $-144 \mathrm{~V}$ occurred during periods of $\leq 4.5$ $\mathrm{keV}$ electron precipitation (assuming no fluxes beyond the energy range of the detector occurred simultaneously). Theoretical secondary electron yields for kapton predict less than one secondary electron per incident electron above 1 to 2 $\mathrm{keV}$ [Katz et al., 1977; Leung et al., 1981], and the yield decreases as the incident electron's energy increases. Coupled with the theoretical expectation that about one tenth of the incident electrons will backscatter [Katz et al., 1977; Prokopenko and Laframboise, 1980], the observation that charging may be due to electrons between 2 and $5 \mathrm{keV}$ seems reasonable since the fluxes at lower energies were relatively small. Of course, the number flux necessary at these energy levels to create a significant charge imbalance is so large that charging is less frequent and severe without higher-energy electron fluxes.

The concept of a material-dependent critical electron temperature (Maxwellian) $\left(E_{t}\right)$ and energy $\left(E_{e}\right)$ being necessary to generate a surface charge imbalance was introduced by $R$ ubin et al. [1978] and is described by Lai et al. [1983] and Katz et al. [1986]. The temperature of a Maxwellian distribution must exceed $E_{t}$ for the incident electrons to outnumber the secondary and backscattered electrons, and at the same time, electrons with energies greater than $E_{e}$ are necessary regardless of temperature for charging to occur. The $E_{t}$ and $E_{e}$ values for kapton range from 0.5 to $0.8 \mathrm{keV}$ and 1.2 to $4.0 \mathrm{keV}$, respectively, depending on the angle of electron incidence and the backscatter and secondary coefficients used [Lai et al., 1983; Katz et al., 1986]. This concept was not specifically addressed in this study because of the Maxwellian dependence, but their theoretical $E_{e}$ range is consistent with the observed energy range discussed in the previous paragraph.

Precipitating electrons below a material-dependent energy level inhibit negative charging by creating multiple secondary electrons. A large percentage of the most severe charging events during solar minimum occurred when over $90 \%$ of the electron flux was at or above $3 \mathrm{keV}$, and many of the least severe charging events during solar minimum had equally large fluxes at higher energies, but also had greater fluxes of lowenergy electrons. Furthermore, on two occasions during solar maximum, the ambient plasma density was lower and the highenergy electron fluxes were greater than during the solar maximum charging event. However, electron fluxes below 1 $\mathrm{keV}$ were also greater and probably prevented a potential from forming. This same feature was found in solar medium events. These results further suggest that 1 to $2 \mathrm{keV}$ is a key electron energy threshold for DMSP charging. This dependence of charging on energetic electron precipitation warrants a consideration of a $K_{p}$ to charging relationship.

Though at least one study of geosynchronous orbit charging has reported a positive correlation between $K_{p}$ level and charging [Mullen et al., 1986], little correlation exists between the $K_{p}$ index and the occurrence or severity of DMSP charging. Relationships between the 3-hour $K_{p}$ values and satellite charging events are shown in Figure 11; the events are denoted by an asterisk for solar minimum charging, "o" for solar medium charging, and " $x$ " for solar maximum charging. The $K_{p}$ values extend through a broad range for a given potential, and the larger $K_{p}$ s generally do not correspond to the greater potentials. The 3 -hour $K_{p}$ index ranged from 0 to 5.0 during the solar minimum period of study, 0 to 7.3 during the solar medium period of study, and 0 to 8.0 during the solar maximum period of study. An interesting feature of the $K_{p}$ during the solar medium events is that the four charging events well into the morning sector (see Figure 8) correspond to the highest $K_{p}$ levels $(4.0-7.3)$ of the solar medium events. The charging near $0600 \mathrm{MLT}$ occurred with a $K_{p}$ of 7.3 ; as already noted, this region was absent of charging during solar minimum when $K_{p} s$ were considerably lower. It is unknown if this feature is purely coincidental. The use of $K_{p}$ as an indicator of high-latitude spacecraft charging is limited because of the nature of the $K_{p}$ (3-hour, global scale). The auroral electrojet $(A E)$ index may be better suited to identify localized currents of short duration, but the $A E$ index is not readily available like the $K_{p}$. Both the $K_{p}$ and $A E$ indexes may 


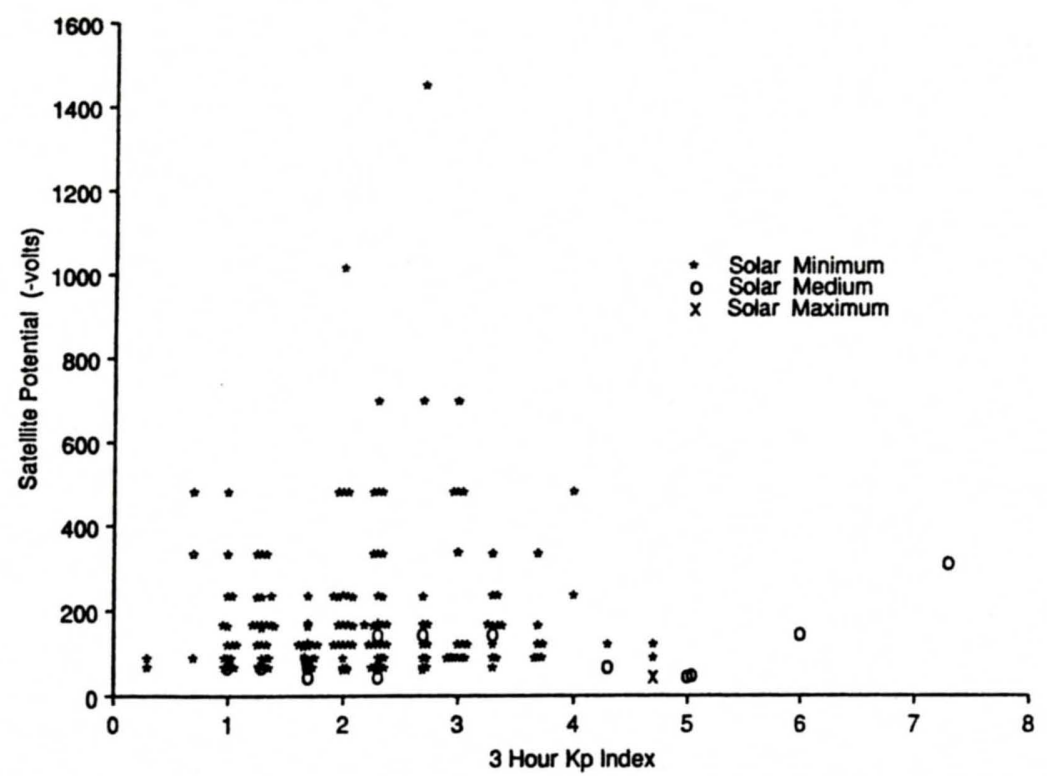

Fig. 11. Scatterplot of the charging potential versus the three hourly $K_{p}$ value for solar minimum (asterisk), solar medium, (o), and solar maximum $(\mathbf{x})$.

show a better correlation if the other current sources were considered.

With polar orbit charging at $840 \mathrm{~km}$ largely a function of ambient plasma density and energetic electron precipitation, the frequency and severity of charging are likely to decrease as altitude decreases. The TDIM predicts densities as low as $5.6 \mathrm{x}$ $10^{3} \mathrm{~cm}^{-3}$ at $300 \mathrm{~km}$ within the polar hole for solar minimum conditions. If $10^{4} \mathrm{~cm}^{-3}$ is indeed an accurate threshold, significant charging is possible even as low as $300 \mathrm{~km}$ and would be limited by the fluxes of energetic electrons. The energy degradation between 800 and $300 \mathrm{~km}$ of precipitating particles is non-trivial, but not to the extent of significantly softening electrons in the 1 to $30 \mathrm{keV}$ energy range. Thus, significant spacecraft charging appears to be possible during solar minimum even near the high-latitude nightside $F 2$ layer peak. Further study is necessary to form conclusions concerning spacecraft charging in this lower region.

\section{CONCLUSION}

The primary causes, frequency, severity, and solar cycle dependence of high-latitude spacecraft charging in low Earth polar orbit $(840 \mathrm{~km})$ have been identified from extensive satellite measurements in the northern hemisphere for winter periods of solar maximum, medium, and minimum. The conditions generating satellite-to-plasma potential differences were analyzed using measured precipitating ion, precipitating electron, and ambient plasma data as well as model ambient plasma results. The conditions which did not cause spacecraft charging were similarly examined.

As a result of these analyses, the following conclusions are drawn: (1) An extreme solar cycle dependence exists; solar minimum conditions generate charging more frequently and with greater magnitude. (2) The chief cause of this dependence is the solar cycle variation of ambient plasma density; the lack of significant charging during solar maximum is due to high ambient plasma densities. (3) Kilovolt charging can occur via natural processes within low Earth orbit. (4) Electrons with energies from 2 to $5 \mathrm{keV}$ contribute to charging though higher energy electrons make greater contributions. Integral number fluxes for electrons $\geq 9.6 \mathrm{keV}$ are normally on the order of $10^{8}$ to $10^{10}$ electrons $/\left(\mathrm{cm}^{2} \mathrm{sec}\right.$ ster $)$ when negative potentials $>100 \mathrm{~V}$ form. (5) A thermal plasma density of $10^{4} \mathrm{~cm}^{-3}$ or less is required for significant charging to occur. (6) Charging is most common in the pre-midnight magnetic frame within the Earth's shadow near $72^{\circ} \mathrm{N}$ magnetic latitude. (7) The $K_{p}$ index is not a strong indicator of charging.

Progress toward further understanding of high-latitude ionospheric charging may be achieved through additional research. Recommended methods include examining charging environments during various solar flux conditions and/or during different seasons. Also, the electron spectra and ambient plasma resulting in different charging levels could be analyzed more closely to fine tune critical energy and density values and to better understand the physical processes which generate electric potentials. An attempt to experimentally quantify all currents, particularly those due to secondary emission, may benefit modeling efforts. Finally, a study of low Earth orbit charging within the southern hemisphere where the TDIM predicts deeper and broader polar density depletions may yield more severe charging.

Acknowledgments. This research was supported by AFOSR-90-0026 grant to Utah State University. The enrollment of T. Frooninckx at Utah State University was sponsored by the United States Air Force Institute of Technology. We are grateful to M. S. Gussenhoven and D. A. Hardy for their insightful discussions. Special thanks to F. J. Rich and J. James for providing extensive DMSP SSIES data and technical expertise, and to $\mathrm{K}$. Lutz and G. Deuel for furnishing the volumes of precipitating particle data. The assistance of $\mathrm{K}$. Marshall, Utah State University, in managing the study's data base is appreciated.

The Editor thanks H. B. Garrett and R. C. Olsen for their assistance in evaluating this paper.

\section{REFERENCES}

DeForest, S. E., Spacecraft charging at synchronous orbit, J. Geophys. Res., 77, 651, 1972.

Garrett, H. B., The charging of spacecraft suffaces, Rev. Geophys., 19, 577, 1981.

Greenspan, M. E., P. B. Anderson, and J. M. Pelagatti, Characteristics of the thermal plasma monitor (SSIES) for the Defense Meteorological Satellite Program (DMSP) spacecraft S8 through S10, Rep. AFGL-TR. 86-0227, Air Force Geophys. Lab., Hanscom Air Force Base, Mass., 1986. 
Gussenhoven, M. S., and E. G. Mullen, Geosynchronous environment for severe spacecraft charging, J. Spacecr. Rockets, 20, 26, 1983.

Gussenhoven, M. S., D. A. Hardy, F. Rich, W. J. Burke, and H.-C. Yeh, High-level spacecraft charging in the low-altitude polar auroral environment, J. Geophys. Res., 90, 11,009, 1985.

Hall, W. N., P. Leung, I. Katz, G. A. Jongeward, J. R. Lilley, Jr., J. E. Nanevicz, J. S. Thayer, and N. J. Stevens, Polar-auroral charging of the Space Shuttle and EVA astronaut, in The Aerospace Environment at High Altitudes and its Implications for Spacecraft Charging and Communications, AGARD-CP-406, p. 34-1, North Atlantic Treaty Organization, France, 1987.

Hardy, D. A., L. K. Schmitt, M. S. Gussenhoven, F. J. Marshall, H.-C. Yeh, T. L. Schumaker, A. Huber, and J. Pantazis, Precipitating electron and ion dectors (SSJ/4) for the block 5D/flights 6-10 DMSP satellites: Calibration and data presentation, Rep. AFGL-TR-84-0317, Air Force Geophys. Lab., Hanscom Air Force Base, Mass., 1984.

Hardy, D. A., M. S. Gussenhoven, and D. Brautigam, A statistical model of auroral ion precipitation, J. Geophys. Res., 94, 370, 1989.

Katz, I., and D. E. Parks, Space shuttle orbiter charging, J. Spacecr. Rockets, 20, 22, 1983.

Katz, I., D. E. Parks, M. J. Mandell, J. M. Harvey, D. H. Brownell, S. S. Wang, and M. Rotenberg, A three dimensional dynamic study of electrostatic charging in materials, NASA Contract. Rep. CR-135256, 1977.

Katz, I., M. Mandell, G. Jongeward, and M. S. Gussenhoven, The importance of accurate secondary electron yields in modeling spacecraft charging, J. Geophys. Res., 91, 13,739, 1986.

Lai, S. T., M. S. Gussenhoven, and H. A. Cohen, The concepts of critical temperature and energy cutoff of ambient electrons in high voltage charging of spacecraft, Eur. Space Agency Spec. Publ., ESA SP-198, $169,1983$.

Leung, M. S., M. B. Tueling, and E. R. Schnauss, Effects of secondary electron emission on charging, Spacecraft charging technology 1980 , NASA Conf. Publ. 2182/AFGL-TR-81-0270, p. 163, 1981.

Mullen, E. G., and M. S. Gussenhoven, High-level spacecraft charging environments near geosynchronous orbit, Rep. AFGL-TR-82-0063, Air Force Geophys. Lab., Hanscom Air Force Base, Mass., 1982.

Mullen, E. G., M. S. Gussenhoven, D. A. Hardy, T. A. Aggson, B. G. Ledley, and E. Whipple, SCATHA survey of high-level spacecraft charging in sunlight, J. Geophys. Res., 91, 1474, 1986.
Olsen, R. C., A threshold effect for spacecraft charging, J. Geophys. Res., 88, 493, 1983.

Olsen, R. C., Record charging events from Applied Technology Satellite 6, J. Spacecr. Rockets, 24, 362, 1987.

Prokopenko, S. M. L., and J. G. Laframboise, High-voltage differential charging of geostationary spacecraft, J. Geophys. Res., 85, 4125, 1980.

Rosen, A., Spacecraft charging: Environment-induced anomalies, $J$. Spacecr. Rockets, 13, 129, 1976.

Rubin, A. G., P. L. Rothwell, and G. K. Yates, Reduction of spacecraft charging using highly emissive surface materials, Proceedings 1978 Symposium on the Effects of the Ionosphere on Space Terrestrial Systems, p. 313, Naval Research Laboratory, Washington, D. C. 1978.

Samir, U., The interaction of large space structures with the near-Earth environment, Proceedings of the Air Force Geophysics Laboratory Workshop on Natural Charging of Large Space Structures in NearEarth Polar Orbit: 14-15 September, 1982, Rep. AFGL-TR-83-0046, Air Force Geophys. Lab., Hanscom Air Force Base, Mass., 1983.

Schumaker, T. L., D. A. Hardy, S. Moran, A. Huber, J. McGarity, and J. Pantazis, Precipitating ion and electron detectors (SSJ/4) for the block 5D/flight 8 DMSP satellite, Rep. AFGL-TR-88-0030, Air Force Geophys. Lab., Hanscom Air Force Base, Mass., 1988.

Schunk, R. W., A mathematical model of the middle and high latitude ionosphere, Pure Appl. Geophys., 127, 255, 1988.

Sojka, J. J., Global scale, physical models of the $F$ region ionosphere, Rev. Geophys., 27, 371, 1989.

Yeh, H.-C, and M. S. Gussenhoven, The statistical electron environment for Defense Meteorological Satellite Program eclipse charging, $J$. Geophys. Res., 92, 7705, 1987.

T. B. Frooninckx, The Air Force Space Forecast Center, Falcon AFB, CO 80912-5000.

J. J. Sojka, Center for Atmospheric and Space Sciences, Utah State University, Logan, UT 84322-4405.

(Received April 4, 1991; revised October 21, 1991; accepted October 21, 1991.) 